\title{
Association between COVID-19 incidence and outcome and national development levels: An ecologic analysis
}

\author{
Chunbao Mo ${ }^{\mathrm{a}, \uparrow}$, Xiaoting $\mathrm{Mo}^{\mathrm{b}, \dagger}$, Tingyu Mai ${ }^{\mathrm{c}, \uparrow}$, Jiansheng Cai ${ }^{\mathrm{b}}$, Xia Xu ${ }^{\mathrm{b}}$, Chuntao Nong ${ }^{\mathrm{b}}$, \\ Dechan Tan ${ }^{\mathrm{c}}$, Shuzhen Liu ${ }^{\mathrm{b}}$, Min $\mathrm{Xu}^{\mathrm{b}}$, Huaxiang $\mathrm{Lu}^{\mathrm{b}}$, Tingyu $\mathrm{Luo}^{\mathrm{c}}$, You $\mathrm{Li}^{\mathrm{c}}$, Jian Qin ${ }^{\mathrm{b}, *}$, \\ Zhiyong Zhang, ${ }^{\mathrm{b}, \mathrm{c} *}$ \\ a Department of Pathophysiology, Faculty of Basic Medical Sciences, Guilin Medical University, Guilin \\ 541004 China \\ b Department of Environmental and Occupational Health, School of Public Health, Guangxi Medical \\ University, Nanning 530021 China \\ ${ }^{c}$ Department of Environmental Health and Occupational Medicine, School of Public Health, Guilin \\ Medical University, Guilin 541004 China
}

*Corresponding authors, e-mail: qinjian@gxmu.edu.cn,rpazz@163.com

$\dagger$ These authors contributed equally to this work.

ABSTRACT: This study aimed to evaluate the association between COVID-19 incidence and outcome and national development level. Spearman correlation analysis method was performed to analyze the relationship between COVID19 incidence, mortality, and recovery rates as of 27 August 2020 and Human Development Index (HDI) and Inequalityadjusted Human Development Index (IHDI) from 177 countries. The pandemic situation varied obviously from country to country, and the most cases mainly concentrated in few countries with very high or high HDI, such as United States and Brazil. The incidence rates of COVID-19 were significantly positively correlated with HDI and IHDI, but the correlation among mortality, recovery rates, HDI, and IHDI were not significant. In the stratified analysis, the incidence rate in countries with low IHDI and the recovery rate in countries with medium IHDI were significantly positively correlated with IHDI. In countries with very high HDI, the mortality rate was significantly positively correlated with HDI. The findings provide new insights into the relationship between COVID-19 and social determinants of health and inspire policy makers to adjust prevention and control strategies for reducing damages caused by this epidemic. Nevertheless, long-term observation and follow-up studies are necessary to be implemented continuously in the future.

KEYWORDS: COVID-19, correlation analysis, HDI, IHDI, ecologic analysis

\section{INTRODUCTION}

The novel coronavirus disease 2019 (COVID-19) is an acute infectious disease caused by the severe acute respiratory syndrome coronavirus 2 (SARSCoV-2) and characterized by respiratory symptoms and fever $[1,2]$. The disease first broke out in Wuhan, China and, then, spread to the whole world, becoming the most serious global public health event in recent years [3]. According to incomplete statistics, a total of 24203260 confirmed cases and 826418 deaths were recorded as of 27 August 2020 worldwide. To control the pandemic of COVID19, national governments have adopted the most stringent prevention and control measures to reduce the flow of population and avoid crowds; and to minimize the risk of virus transmission by imple- menting lockdown of cities, school closures, factory shutdowns, travel restrictions, etc. Although some governments have controlled the pandemic effectively in their own countries, the situation of the COVID-19 pandemic is still not optimistic from the global perspective.

Previous serious public health events showed that the impact of outbreak on poor and vulnerable groups is often disproportionate $[4,5]$. In particular, social vulnerability and inequality in this outbreak have contributed to the morbidity and adverse outcomes of COVID-19 in many ways, thereby facilitating the spread and intensifying severity of this disease to a certain extent [6]. The outbreak has promoted the occurrence of economic crises, increased unemployment, and weakened the social welfare safety net; in this regard, the poor, which 
are socially and economically disadvantaged, face greater health risks [5]. Therefore, incorporating the social determinant of health into the COVID-19 pandemic research is necessary. Most previous studies on the morbidity and mortality of the COVID19 pandemic influenced by socio-economic factors focused on the individual or social level [6-8]; no national study to date has evaluated the association between social determinants and COVID-19.

In this ecological study, we analyzed the correlation between the incidence and outcome of COVID-19 and the Human Development Index (HDI) and Inequality-adjusted Human Development Index (IHDI) from 177 countries to determine the relationship of national development and pandemic situation. The findings would provide new insights into the relationship between COVID-19 and social determinants of health and inspire policy makers to adjust prevention and control strategies for reducing damages caused by the epidemic.

\section{MATERIALS AND METHODS}

\section{Data resource}

Since the outbreak of COVID-19, numerous institutions have released real-time epidemic data. In this study, data, including cumulative number of confirmed cases, deaths, and recovered cases, were obtained from the Website of Johns Hopkins University as of 27 August 2020 [9]. HDI and IHDI data were obtained from the Human Development Report released by the United Nations Development Program (UNDP) [10], which is released annually. Given that the data of 2019 have not yet been released and are relatively stable in recent years, HDI and IHDI data published in 2018 would be used in this study. The demographic data of each country were obtained from the World Health Organization (WHO) website [11]. All the data in this research were public and did not involve any personal information, so ethical approval was not required.

\section{HDI and IHDI}

HDI data have been published by the UNDP since 1990 and used to measure the level of socioeconomic development of countries or regions. HDI is a composite index of health, education, and income ranging from 0 to 1 . Health index is based on life expectancy at birth, education index is calculated based on the mean years of schooling and expected years of schooling, and GNI per capita (per capita gross national income) is used to calculate the income index. Specific calculation formulas and explanations can be referenced from technical notes [12]. According to HDI, countries can be divided into four categories as follows: countries with very high HDI (HDI $\geqslant 0.800)$, high HDI $(0.799 \geqslant$ HDI $\geqslant 0.700)$, medium HDI $(0.699 \geqslant$ HDI $>0.550)$, and low HDI (HDI $\leqslant 0.550)$.

The effects of inequality among various countries are not considered in HDI; therefore, the applicability of this index is often questioned. Inequalityadjusted Human Development Index (IHDI), which is based on a distribution-sensitive class of composite indices proposed by Foster, Lopez-Calva, and Sleekly (2005), completely considers statistical biases caused by social inequality [12]. IHDI explains the inequality of HDI dimensions. IHDI equals HDI when no inequality exists across people but falls below the HDI when inequality rises.

\section{Statistical analysis}

Spearman correlation analysis was used to explore the correlation of the incidence, mortality, and recovery rates of COVID-19 to HDI and IHDI. The strength of the correlation between two variables was expressed by Spearman correlation coefficient $\left(r_{s}\right): r_{s}>0$, positive correlation; $r_{s}<0$, negative correlation; and $r_{s}=0$, irrelevant. The significance level of $p<0.05$ was considered. We first analyzed the correlation between overall incidence, mortality, and recovery rate of COVID-19 and HDI and IHDI. Countries were grouped according to the values of HDI and IHDI, and correlation analysis was conducted again. The above methods were performed by SPSS statistical software (version 24.5 for Windows software).

\section{RESULTS}

\section{Prevalence of COVID-19}

At present, COVID-19 has spread globally and is distributed in 188 countries or regions. Countries with more than 1 million cumulative confirmed cases include the United States, Brazil, and India, and more than half of the total confirmed cases worldwide are found in these countries. In the semilogarithmic coordinates in Fig. 1, the Y-axis is the logarithmic scale, and countries are arranged in descending order by HDI on the X-axis. The figure exhibits the prevalence of COVID-19 in various countries, which were divided into four categories according to HDI. In general, the prevalence of the situation varied in different countries. The number of cumulative confirmed cases was above 100000 in countries with very high or high HDI, but this num- 


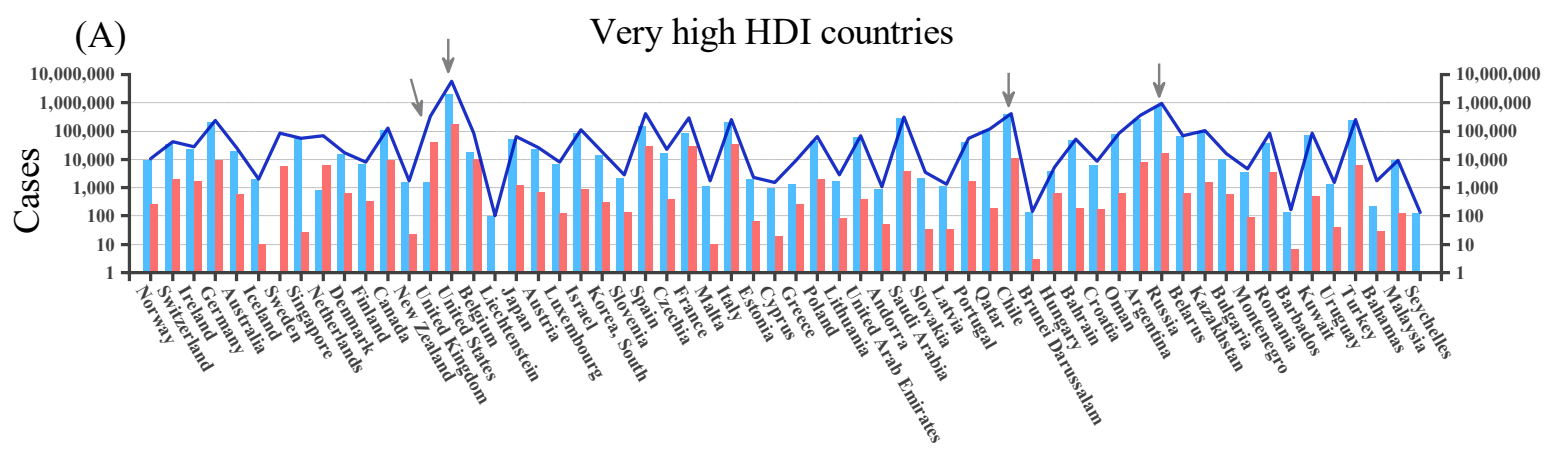

(B)
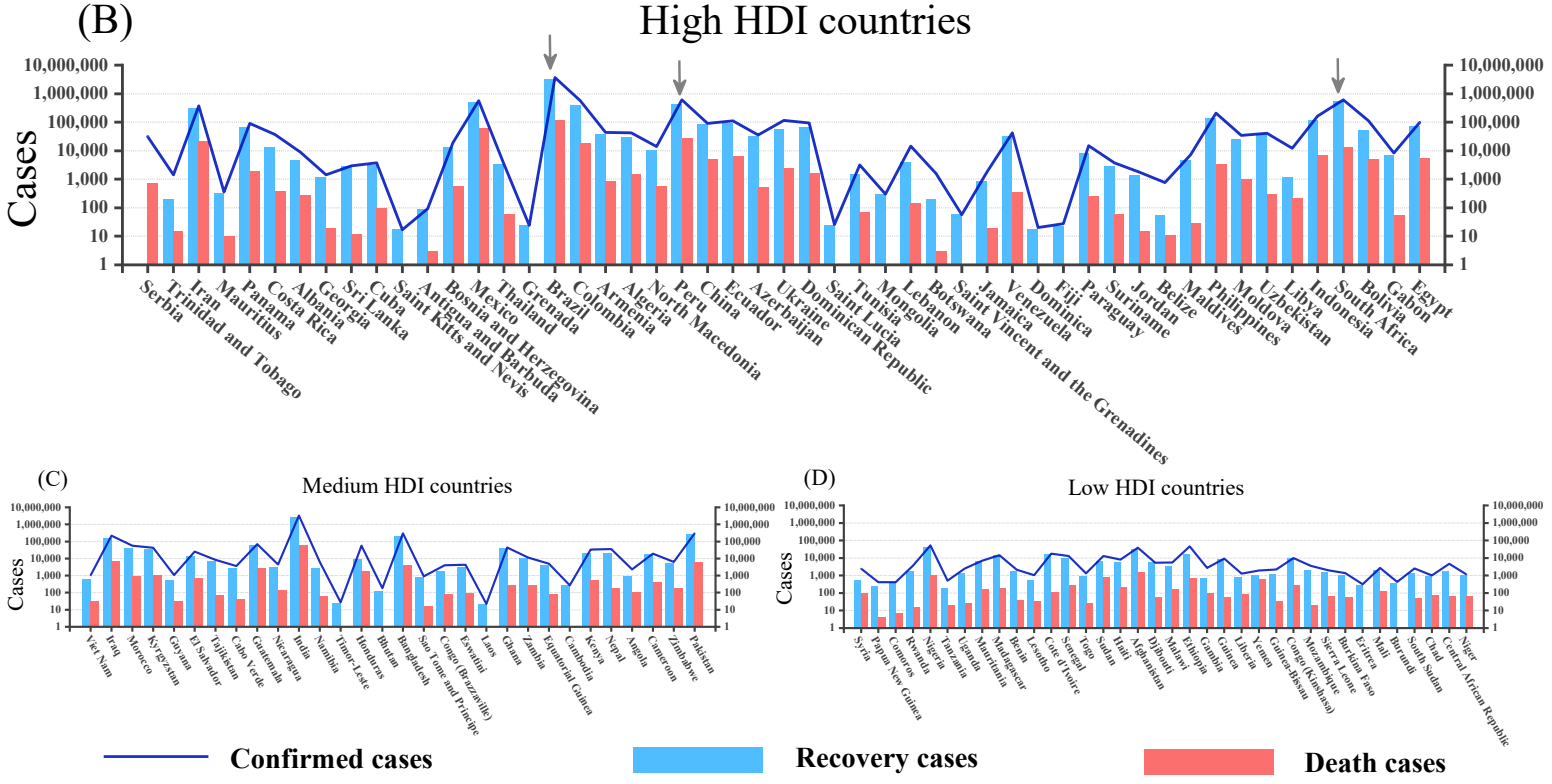

Fig. 1 Prevalence of COVID-19 in various countries. (A-D) Four categories of countries development according to HDI: the Y-axis was logarithmic scale, and countries were arranged in descending order by HDI on the X-axis.

ber was low in countries with medium or low HDI. Cumulative confirmed cases, deaths, and recovered cases were mainly distributed in few countries with very high HDI (e.g., United States, Russian, Chile, and United Kingdom) and high HDI (e.g., Brazil, South Africa, and Peru). The number of cases in the other countries was relatively small.

\section{Correlation analysis}

We analyzed the correlation of the incidence, mortality, and recovery rates of COVID-19 to HDI and IHDI. Overall, the incidence rate of COVID19 was significantly positively correlated with HDI and IHDI, and the correlation was strong. This finding suggested that the incidence of COVID-19 increased with increasing HDI and IHDI. However, the mortality and recovery rates of COVID-19 were not positively correlated with HDI and IHDI (Fig. 2, Table 1).
Table 1 Correlation analysis results and correlation coefficients between COVID-19 incidence, mortality, and recovered rates and HDI and IHDI.

\begin{tabular}{|c|c|c|c|c|c|c|}
\hline & \multicolumn{2}{|c|}{ Incidence rate } & \multicolumn{2}{|c|}{ Mortality rate } & \multicolumn{2}{|c|}{ Recovery rate } \\
\hline & $r_{s}$ & $p$ & $r_{s}$ & $p$ & $r_{s}$ & $p$ \\
\hline HDI & 0.507 & $<0.001^{*}$ & 0.118 & 0.119 & 0.146 & 0.054 \\
\hline IHDI & 0.492 & $<0.001^{*}$ & 0.156 & 0.060 & 0.123 & 0.140 \\
\hline
\end{tabular}

" Statistically significant.

Given that different countries have different levels of development, the relationship of the incidence, mortality, and recovery rates of COVID-19 to HDI and IHDI should be further analyzed at the same level of development. We divided all countries into four hierarchies based on HDI and IHDI values and performed correlation analysis. The incidence rate in the low IHDI group and the recovery rate in 
(A)

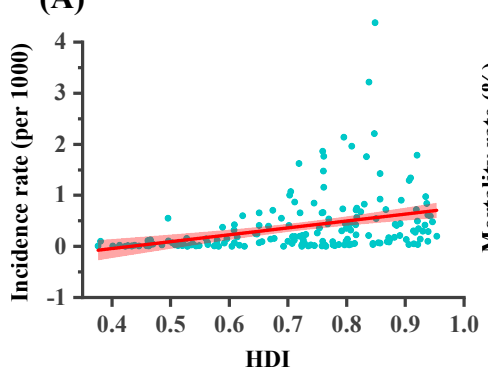

(D)

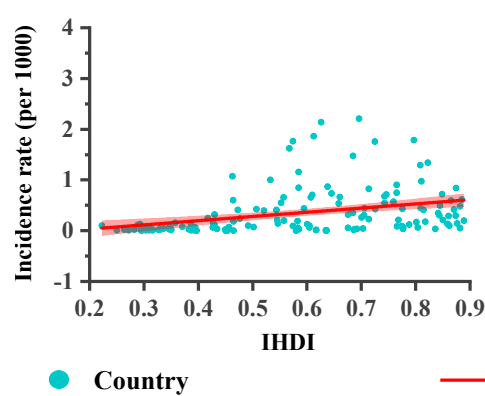

(B)

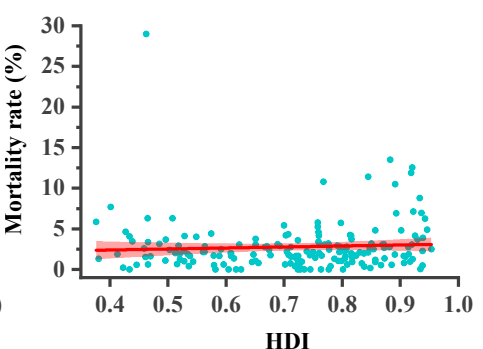

(E)

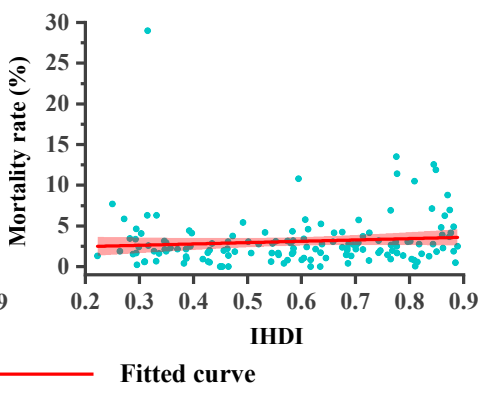

(C)

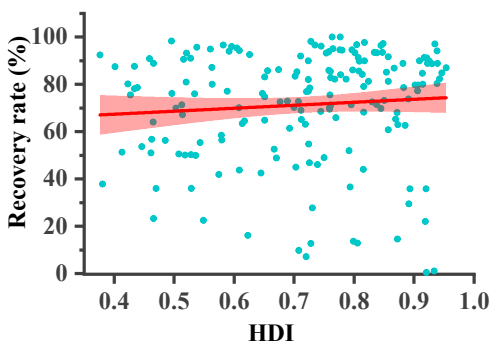

(F)

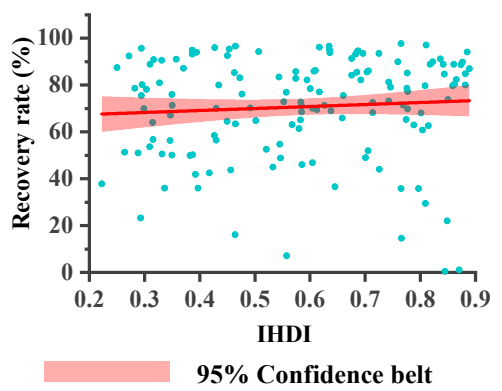

Fig. 2 Scatter plots and linear fit curve of countries' incidence, mortality, and recovered rates and HDI and IHDI.

Table 2 Stratified analysis and correlation coefficient between COVID-19 incidence, mortality, and recovery rates and HDI and IHDI.

\begin{tabular}{lrcrcrrr}
\hline & \multicolumn{2}{c}{ Incidence rate } & \multicolumn{2}{r}{ Mortality rate } & \multicolumn{2}{r}{ Recovery rate } \\
\cline { 2 - 7 } & $r_{s}$ & $p$ & $r_{s}$ & $p$ & $r_{s}$ & $p$ \\
\hline HDI & & & & & & \\
Very high & 0.020 & 0.880 & 0.297 & $0.021^{*}$ & -0.094 & 0.477 \\
High & -0.014 & 0.924 & 0.141 & 0.324 & 0.238 & 0.096 \\
Medium & 0.369 & $0.045^{*}$ & 0.205 & 0.276 & -0.235 & 0.211 \\
Low & 0.174 & 0.312 & -0.171 & 0.320 & -0.145 & 0.399 \\
\hline IHDI & & & & & & \\
Very high & -0.063 & 0.761 & 0.215 & 0.291 & 0.221 & 0.288 \\
High & -0.173 & 0.430 & -0.027 & 0.902 & -0.163 & 0.457 \\
Medium & -0.075 & 0.652 & $<0.001$ & 0.999 & 0.488 & $0.002^{*}$ \\
Low & 0.491 & $<0.001^{*}$ & -0.155 & 0.241 & -0.009 & 0.947 \\
\hline
\end{tabular}

* Statistically significant.

the medium IHDI group were significantly positively correlated with IHDI $\left(r_{s}=0.491, p<0.001\right.$; and $r_{s}=0.488, p=0.002$, respectively). In countries with very high HDI, the mortality rate was significantly positively correlated with HDI $\left(r_{s}=0.297\right.$, $p=0.021$ ). In the other groups, the incidence, mortality, and recovery rates were not significantly correlated with HDI or IHDI (Fig. 3, Table 2).

\section{DISCUSSION}

The outbreak of COVID-19 is the most serious global public health event in recent decades [13] and has greater scale and influence compared with the epidemic of SARS, MERS, and flu [14]. The impact of this outbreak will expand and will not reach its peak in a short time because considerable uncertainty exists with regard to the reach of the virus, the medical supply reserve, the efficacy of the policy response, and other aspects. Socio-economic factors are significant for the outbreak and have received extensive attention. In this study, we analyzed the correlation of the incidence, mortality, and recovery rates of COVID-19 to HDI and IHDI from 177 countries to determine the relationship of national development and the pandemic situation. The main findings were as follows. (1) The pandemic situation varied obviously from country to country, and the highest number of cases mainly concentrated in countries with very high or high HDI, such as the United States and Brazil. (2) The incidence rates of COVID-19 were significantly positively correlated with HDI and IHDI, but the correlation between mortality and recovery rates and HDI and IHDI were not significant. (3) In the stratified analysis, the incidence rate in countries with low IHDI and the recovery rate in countries with medium IHDI were significantly positively correlated with IHDI. In countries with very high HDI, the mortality rate was significantly positively correlated with HDI. These findings provided new evidence for the association between COVID-19 pandemic and socio-economic factors and references for decision-makers to adjust prevention strategies.

HDI was initially utilized to measure the socio- 
(A) Very high HDI countries
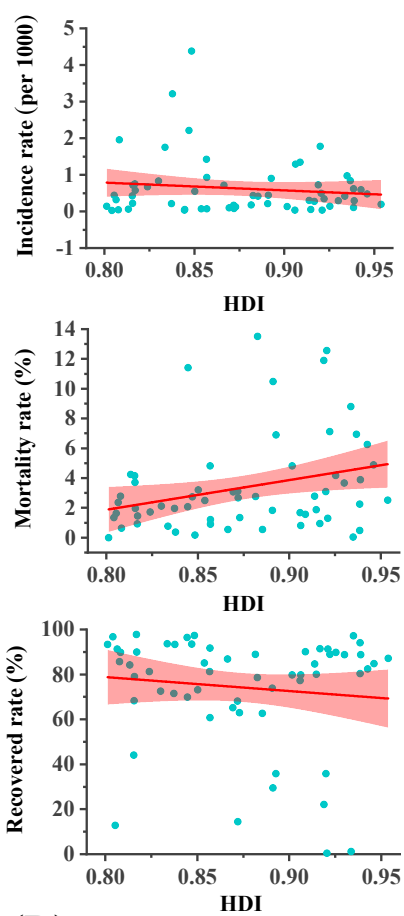

(B) Very high IHDI countries
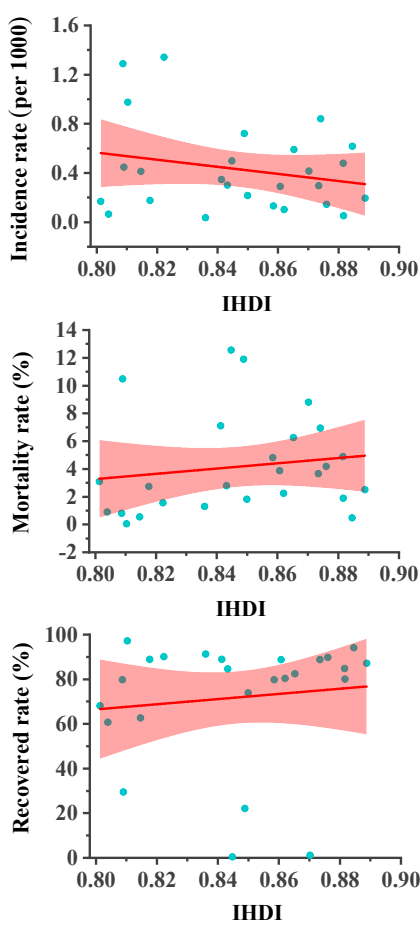

Country
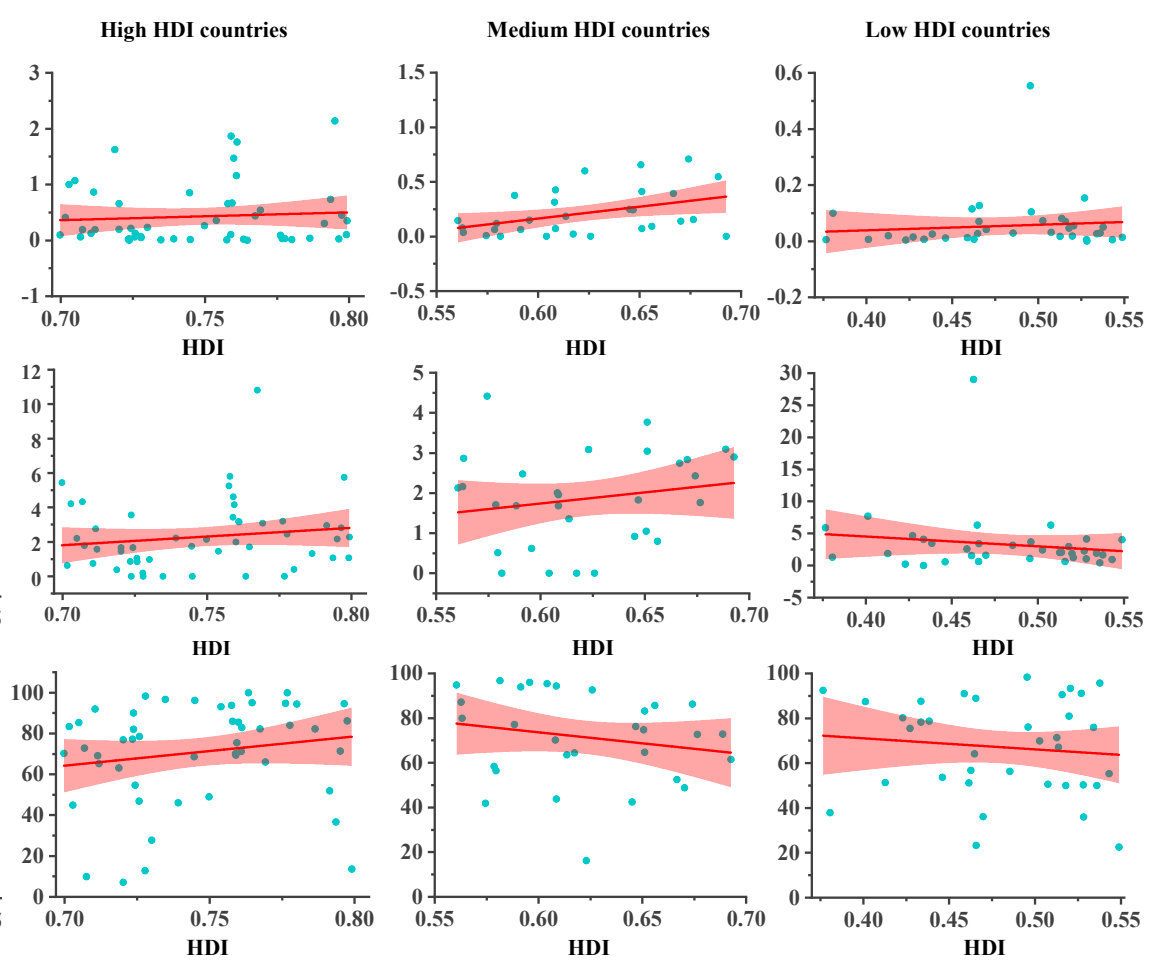

High IHDI countries

Medium IHDI countries
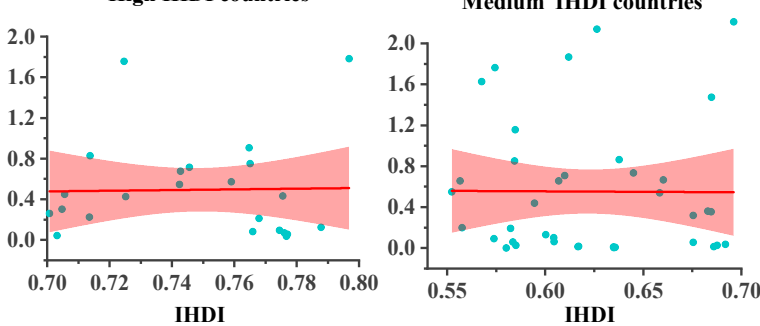

Low IHDI countries
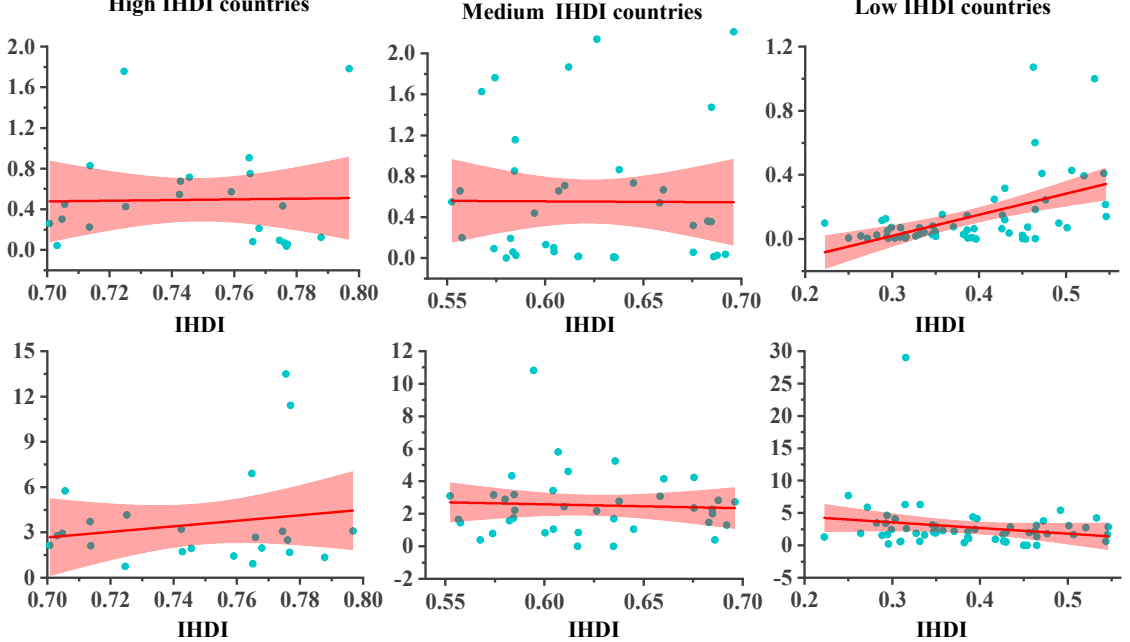

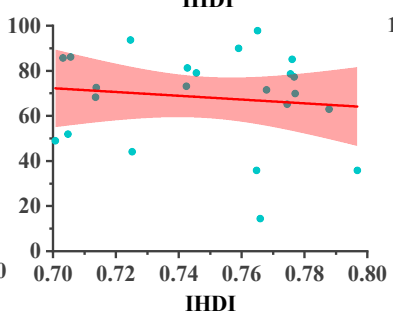

Fitted curve

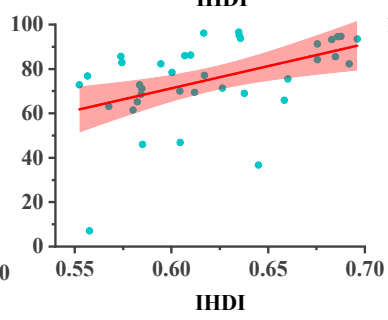

IHDI

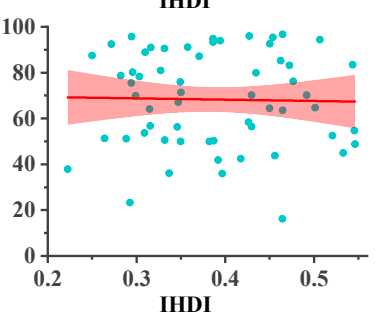

95\% Confidence belt

Fig. 3 Scatter plots and linear fit curve of countries' incidence, mortality, and recovered rates and HDI and IHDI, stratified based on different levels of development. 
economic development of member states in the United Nations, and its index significance on comprehensive measurement is accepted worldwide and recognized as a beneficial supplement to GDP, GNP, and other indicators. Advantages of this index are as follows: (1) its meaning is definite and data acquisition, calculation and comparison are easy; (2) it is applicable to various groups and can reflect discrepancies in income distribution, gender, regional distribution and ethnic groups [15]. Although HDI is a vital and comprehensive indicator for measuring the socio-economic development level of countries or regions, its kernel still emphasizes that "people and their capabilities should be the ultimate criteria for assessing the development of a country, not economic growth alone", and that the average achievement in key aspects of human development includes a long and healthy life, being knowledgeable, and a decent standard of living [16]. In brief, HDI measures the level of human development and reflects the social progress that provides a new perspective for people to evaluate social development. Previous experiences remind us that epidemics are not only a medical issue, but also a social problem. Factors, such as income inequality, educational background, and availability of healthcare services, may affect the incidence and outcome of infectious diseases [17]. Therefore, as an intuitive indicator generalizing the above factors, HDI should be considered to explore the association between national development level and COVID-19 incidence and outcome.

In general, HDI is considered to be negatively correlated with disease morbidity or mortality; this conclusion has been confirmed by many previous studies [18-21]. The incidence, mortality, and recovery rates of COVID-19 increased with increasing HDI and IHDI, suggesting a positive correlation between the pandemic situation and national development, consistent with the results of the research by Sebastiano Gangemi, et al [8]. The contradicting findings may be explained as follows. Compared with some chronic diseases (e.g., cervical cancer, hypertension, obesity, etc.) and infectious diseases (e.g., TB, AIDS, malaria, etc.), emerging infectious diseases (EIDs) cannot be easily prevented and controlled due to uncertainty of outbreak, limitation of knowledge of the disease, insufficient herd immunity, lag of therapy, and so on [22,23]. Moreover, prevention systems built on existing knowledge of infectious diseases may not be always efficacious. Therefore, countries will face the risk of spreading COVID-19. An objective fact is that developed countries are characterized by high urbanization rates, dense urban population, sound transportation networks, and convenient commuting conditions, thereby facilitating the speed and breadth of virus spread [24]. Previous studies suggested the significant positive correlation between the number of cases and domestic traffic [25]. The United States, Canada, and other countries reported the cases to be related to domestic and international travel [26,27]. In addition, Maogui Hu, et al [28] assessed the risk of COVID-19 transmission in train by using modelling study. The personal income level is generally poor in developing countries compared with that in developed countries; people with poor economic conditions are more likely to possess poor social network and low social support. Hence, external and non-home-based relationships are less established in low-income groups; however, the group with higher economic status may participate in more social activities and spend more time to stay in densely populated places, such as business activities, parties, shopping, concerts, etc.; the difference increases with socio-economic status [29]. Thus, population clustering provides favorable conditions for viral transmission.

Another explanation may be related to higher rates of screening, detection, and treatment in developed countries, while in less developed countries, reliable case screening and timely treatment are difficult to ensure [30,31]. Early identification of virus carriers and patients and implementing isolation and treatment measures to stop viral spread are crucial for epidemical prevention and control. These processes require the government to provide a large amount of financial funds and medical supply supports. For example, viral nucleic acid screening of high-risk groups, disinfection and management of contaminated sites, therapy of confirmed patients, medical insurance fund reserve, and so on need to expend a high amount of government funds. According to statistics, the Chinese and the United States governments have successively invested 175.6 billion yuan (RMB) and 50 billion US dollars [32], respectively, in epidemic prevention. However, these necessary costs make it difficult for low-developing countries to sustain. In addition, the poor medical conditions, lack of materials, and other problems in these counties cause difficulties in identifying the infection sources and diagnosis of suspected cases, thereby, obviously reducing the possibility of case detection. Consequently, the "iceberg" phenomenon of COVID-19 may be more prominent in low-developing countries [30]. 
In this study, overall, the COVID-19 mortality and recovery rates were not significantly associated with HDI and IHDI, which may be related to the characteristics of the disease. At present, no specific treatment, drugs, monoclonal antibodies (mAbs), or vaccines exist for SARS-CoV-2 [33]. Based on in vitro experiments and clinical treatment experience of SARS-COV, MERS-CoV, and other coronaviruses, treatment is essentially supportive and symptomatic. In addition, most cases displayed no, mild, or moderate symptoms, accounting for about $85 \%$ of all cases; as such, special treatment is not necessary for them except for maintaining good hydration and nutrition, controlling fever and cough, and isolation [34]. As a result, the recovery rate is generally high in each country. As the outbreak is not over yet, the number of cases is being updated daily, so cumulative confirmed cases, as the denominator, strongly influenced the mortality and recovery rates. We calculated Spearman correlation coefficient $\left(r_{s}\right)$ between mortality and recovery rates and HDI and IHDI in different periods and found that the correlation coefficient and significance decreased gradually. Therefore, continuous observations and researches are necessary.

In the stratified analysis, the incidence, mortality, and recovery rates of COVID-19 were not significantly correlated with HDI and IHDI at most levels, but only weakly correlated with low, medium, and very high HDI levels. This phenomenon may be caused by statistical error; therefore, the following limitations of the study should be improved. First, the pandemic is not over yet, and case data are being updated every day; thus, data involved in this study do not wholly reflect the COVID-19 pandemic, and the conclusion may be skeptical. Second, stratified heterogeneity may potentially affect the result; hence, more and more detailed data should be collected for further analysis and calculation. For instance, mortality is regarded as crude among many available calculations, so age-adjusted mortality may be more appropriate. Third, this work is an ecological research; as such, avoiding bias caused by ecological fallacy is difficult [35]. Finally, we did not consider the different impacts of national public health strategies, the efficacy of government leadership, social hot spots, regional culture and religion, and other socio-economic factors on the COVID-19 spread. Long-term observation and follow-up studies are necessary to be implemented continuously in the future so we can identify the relationship between national development and COVID-19 pandemic situation.
A study using the ARIMA model predicts that countries with the highest number of COVID-19 cases will appear between December 2020 and April 2021. In addition, the study predicts that if current preventive measures are completely relaxed, a second pandemic rebound could occur within a year [36]. We must consider the current uncertainty of the COVID-19 pandemic, as well as an increasingly interconnected and complex world that requires a flexible and resilient response to future unexpected events and circumstances.

\section{Data availability statement}

The data that support the findings of this study were derived from the following resources available in the public domain: Johns Hopkins Coronavirus Resource Center (https: //coronavirus.jhu.edu/map.html), United Nations Development Program (http://hdr.undp.org/en/ content/human-development-report-2019), and United Nations (https://unstats.un.org/unsd/ demographic-social/index.cshtml).

Acknowledgements: The authors would like to thank the Johns Hopkins Coronavirus Resource Center, United Nations Development Program (UNDP) and United Nations for the various reports and the latest data published. The views expressed in this paper are those of the authors and do not necessarily represent those of their institutions. This study was supported by Innovation Project of Guangxi Graduate Education (No. YCSW2020230).

\section{REFERENCES}

1. Paules CI, Marston HD, Fauci AS (2020) Coronavirus infections-more than just the common cold. $J$ Am Med Assoc 323, 707-708.

2. Huipeng G, Xiufen W, Xiangning Y, Gong X, Chengzhi W, Tianci D, Qiongjing Y, Xiangcheng X (2020) The epidemiology and clinical information about COVID19. Eur J Clin Microbiol 39, 1011-1019.

3. Sohrabi C, Alsafi Z, O’Neill N, Khan M, Kerwan A, Al-Jabir A, Iosifidis C, Agha R (2020) World Health Organization declares global emergency: A review of the 2019 novel coronavirus (COVID-19). Int J Surg 76, 71-76.

4. Elissa MA, Stanley JS (2020) COVID-19 and the impact of social determinants of health. Lancet Resp Med 8, 659-661.

5. Ahmed F, Ahmed N, Pissarides C, Stiglitz J (2020) Why inequality could spread COVID-19. Lancet Public Health 5, e240.

6. Nayak A, Islam SJ, Mehta A, Ko Y-A, Patel SA, Goyal A, Sullivan S, Lewis TT, et al (2020) Impact of social vulnerability on COVID-19 incidence and out- 
comes in the United States. medRxiv, ID 20060962. [Preprint]

7. Liotta G, Marazzi MC, Orlando S, Palombi L (2020) Is social connectedness a risk factor for the spreading of COVID-19 among older adults? The Italian paradox. PLoS One 15, e0233329.

8. Gangemi S, Billeci L, Tonacci A (2020) Rich at risk: Socio-economic drivers of COVID-19 pandemic spread. Clin Mol Allergy 18, ID 12.

9. Johns Hopkins Coronavirus Resource Center. Available at: https://coronavirus.jhu.edu/map.html.

10. McGillivray M, White H (1993) Measuring development? The UNDP's human development index. $J$ Int Dev 5, 183-192.

11. United Nations. Demographic and Social Statistics. Available at: https://unstats.un.org/unsd/ demographic-social/index.cshtml.

12. UNDP (2019) Beyond income, beyond averages, beyond today: Inequalities in human development in the 21st century. Human Development Report 2019, NY, USA. Available at: http://hdr.undp.org/ en/content/human-development-report-2019.

13. Peeri NC, Shrestha N, Rahman MS, Zaki R, Tan Z, Bibi S, Baghbanzadeh M, Aghamohammadi N, et al (2020) The SARS, MERS and Novel Coronavirus (COVID-19) epidemics, the newest and biggest global health threats: what lessons have we learned? Int $J$ Epidemiol 49, 717-726.

14. Mo C, Tan D, Mai T, Bei C, Qin J, Pang W, Zhang Z (2020) An analysis of spatiotemporal pattern for COIVD-19 in China based on space-time cube. $J$ Med Virol 92, 1587-1595.

15. Noorbakhsh F (2002) Human development and regional disparities in Iran: a policy model. $J$ Int Dev 14, 927-949.

16. Scherbov S, Gietel-Basten S (2020) Measuring inequalities of development at the sub-national level: From the human development index to the human life indicator. PLoS One 15, e0232014.

17. Ataey A, Jafarvand E, Adham D, Moradi-Asl E (2020) The relationship between obesity, overweight, and the human development index in World Health Organization eastern Mediterranean region countries. J Prev Med Public Health 53, 98-105.

18. Goodarzi E, Beiranvand R, Darvishi I, NaghibzadehTahami A, Bechashk SM, Naemi H, Khazaei Z (2020) Geographical distribution of falciparum malaria in the world and its relationship with the human development index (HDI): countries based on the WHO report in 2017. J Public Health (Berl), 1-10.

19. Khazaei Z, Sohrabivafa M, Mansori K, Naemi H, Goodarzi E (2019) Incidence and mortality of cervix cancer and their relationship with the human development index in 185 countries in the world: An ecology study in 2018. Adv Hum Biol 9, ID 222.

20. Hassanipour-Azgomi S, Mohammadian-Hafshejani A, Ghoncheh M, Towhidi F, Jamehshorani S, Sale- hiniya $H$ (2016) Incidence and mortality of prostate cancer and their relationship with the Human Development Index worldwide. Prostate Int 4, 118-124.

21. Dye C, Lonnroth K, Jaramillo E, Williams BG, Raviglione $M$ (2009) Trends in tuberculosis incidence and their determinants in 134 countries. B World Health Organ 87, 683-691.

22. Ellwanger JH, Kaminski VDL, Chies JAB (2019) Emerging infectious disease prevention: Where should we invest our resources and efforts? J Infect Public Heal 12, 313-316.

23. Morens DM, Folkers GK, Fauci AS (2004) The challenge of emerging and re-emerging infectious diseases. Nature 430, 242-249.

24. Wu JT, Leung K, Leung GM (2020) Nowcasting and forecasting the potential domestic and international spread of the 2019-nCoV outbreak originating in Wuhan, China: A modelling study. Lancet 395, 689-697.

25. Zhao S, Zhuang Z, Ran J, Lin J, Yang G, Yang L, He D (2020) The association between domestic train transportation and novel coronavirus (2019-nCoV) outbreak in China from 2019 to 2020: A datadriven correlational report. Travel Med Infect Di 33, ID 101568.

26. Silverstein WK, Stroud L, Cleghorn GE, Leis JA (2020) First imported case of 2019 novel coronavirus in Canada, presenting as mild pneumonia. Lancet 395, ID 734.

27. Schwartz KL, Murti M, Finkelstein M, Leis JA, Fitzgerald-Husek A, Bourns L, Meghani H, Saunders A, et al (2020) Lack of COVID-19 transmission on an international flight. Can Med Assoc J 192, e410.

28. Hu M, Lin H, Wang J, Xu C, Tatem AJ, Meng B, Zhang X, Liu Y, et al (2020) Risk of coronavirus disease 2019 transmission in train passengers: an epidemiological and modeling study. Clin Infect Dis 72, 604-610.

29. Weyers S, Dragano N, Mobus S, Beck EM, Stang A, Mohlenkamp S, Jockel KH, Erbel R, et al (2008) Low socio-economic position is associated with poor social networks and social support: results from the Heinz Nixdorf Recall Study. Int J Equity Health 7, 13.

30. Kobia F, Gitaka J (2020) COVID-19: Are Africa's diagnostic challenges blunting response effectiveness? AAS Open Res 3, ID 4.

31. Anjum FR, Anam S, Rahman SU (2020) Novel Coronavirus disease 2019 (COVID-19): New challenges and new responsibilities in developing countries. Hum Vaccines Immunother 16, 2370-2372.

32. The State Council of the People's Republic of China (2020) Report on the Implementation of China's Fiscal Policy in the First Half of 2020. Available at: http://www.gov.cn/xinwen/2020-08/ 06/content_5532865.htm. [in Chinese]

33. Wu Z, McGoogan JM (2020) Characteristics of and important lessons from the Coronavirus Disease 2019 (COVID-19) outbreak in China: Summary of 
a report of 72314 cases from the Chinese Center for Disease Control and Prevention. J Am Med Assoc 323, 1239-1242.

34. Madabhavi I, Sarkar M, Kadakol N (2020) COVID19: A review. Monaldi Arch Chest Dis 90, 248-258.

35. Morgenstern H (1982) Uses of ecologic analysis in epidemiologic research. Am J Public Health 72,
1336-1344.

36. Malki Z, Atlam ES, Ewis A, Dagnew G, Alzighaibi AR, ELmarhomy G, Elhosseini MA, Hassanien AE, et al (2020) ARIMA models for predicting the end of COVID-19 pandemic and the risk of second rebound. Neural Comput Appl, 1-20. 